\title{
A hybrid GMRES and TV-norm based method for image restoration
}

\author{
D. Calvetti ${ }^{a}$, B. Lewis ${ }^{b}$ and L. Reichel ${ }^{c}$ \\ ${ }^{a}$ Department of Mathematics, Case Western Reserve University, Cleveland, OH 44106 \\ ${ }^{b}$ Rocketcalc, LLC, Richfield, OH 44286 \\ ${ }^{c}$ Department of Mathematics, Kent State University, Kent, OH 44242
}

\begin{abstract}
Total variation-penalized Tikhonov regularization is a popular method for the restoration of images that have been degraded by noise and blur. The method is particularly effective, when the desired noise- and blur-free image has edges between smooth surfaces. The method, however, is computationally expensive. We describe a hybrid regularization method that combines a few steps of the GMRES iterative method with total variation-penalized Tikhonov regularization on a space of small dimension. This hybrid method requires much less computational work than available methods for total variation-penalized Tikhonov regularization and can produce restorations of similar quality.
\end{abstract}

Keywords: Regularization, image restoration, GMRES, total variation

\section{INTRODUCTION}

We consider the restoration of images that have been degraded by noise and blur, where the desired noise- and blur-free image has edges between smooth surfaces. The blurring of an image by spatially invariant blur can be modeled by a convolution

$$
g(\xi, \eta)=\int_{\mathbf{R}^{2}} h\left(\xi-\xi^{\prime}, \eta-\eta^{\prime}\right) f\left(\xi^{\prime}, \eta^{\prime}\right) d \xi^{\prime} d \eta^{\prime},
$$

where the input and output functions $f$ and $g$ represent the original and blurred images, respectively. The smooth kernel $h$ is referred to as a point spread function. The problem of determining the input function $f$, given the output function $g$, in integral equations of the form (1) is ill-posed, because the solution $f$, when it exists, does not depend continuously on $g$.

We assume that the functions $f, g$, and $h$ in (1) are identically zero outside the unit square $\Omega \subset \mathbf{R}^{2}$, and that equation (1) is discretized by a regular $m \times m$ mesh to yield a finite system of linear (algebraic) equations

$$
A x=b, \quad A \in \mathbf{R}^{m^{2} \times m^{2}}, \quad x, b \in \mathbf{R}^{m^{2}},
$$

where the vector $x$ contains the row-wise ordering of the nonnegative, real pixel values of the discrete image corresponding to $f$ in equation (1). The matrix $A$ is the blurring matrix formed by the entries of the discretized point spread function and the discretization of the integral operator. The vector $b$ contains row-wise ordered pixel values of the blurred discretized image associated with $x$. The matrix $A$ has many singular values of different orders of magnitude close to the origin; thus, $A$ is of ill-determined rank. We refer to linear systems of equations (2) with a matrix of ill-determined rank as discrete ill-posed problems. A recent discussion on many numerical methods for the solution of discrete ill-posed problems is provided by Hansen. ${ }^{8}$ In many applications, a discrete point spread function is determined empirically, and the matrices $A$ that arise often are nonsymmetric.

In typical applications the vector $b$ in $(2)$ is not available. Instead, the vector

$$
\tilde{b}=b+d
$$

E-mail: D.C.: dxc57@po.cwru.edu, B.L.: blewis@rocketcalc.com, L.R.: reichel@math.kent.edu 
is known. The entries of the vector $d \in \mathbf{R}^{n}$ contain measurement and transmission errors. We refer to $d$ as "noise." Thus, the vector $\tilde{b}$ represents an available discretized image that is contaminated by blur and noise.

The desired, but unknown, discretized image $x$ solves the linear systems of equations (2) with unknown right-hand side vector $b$. We seek to determine an approximation of $x$ by computing an approximate solution of the linear system of equations

$$
A \tilde{x}=\tilde{b}, \quad \tilde{x}, \tilde{b} \in \mathbf{R}^{m^{2}}
$$

with known right-hand side (3). For notational simplicity, we assume for the remainder of the paper that the matrix $A$ is nonsingular, and we denote the unique solution of (4) by $\tilde{x}=A^{-1} \tilde{b}$. Note that since the matrix $A$ has many singular values close to the origin, it is severely ill-conditioned. Because of this, and due to the noise $d$ in the available right-hand side $\tilde{b}$, the solution $\tilde{x}$ of (4) is typically a poor approximation of the solution $x$ of $(2)$.

Regularization methods produce solutions $\tilde{x}_{\alpha}$ of a problem different from, but related to (4). The solution $\tilde{x}_{\alpha}$ is referred to as a regularized solution of (4), and it depends on $A, \tilde{b}$, the regularization method, and the value of the regularization parameter $\alpha$ (and sometimes on the values of additional parameters as well). Regularization methods often produce much better approximations of the desired vector $x$ than the solution $\tilde{x}$ of (4).

One of the most popular regularization methods is Tikhonov regularization. The regularized solution $\tilde{x}_{\alpha}$ produced by this regularization method satisfies the minimization problem

$$
\tilde{x}_{\alpha}=\min _{x \in \mathbf{R}^{m^{2}}}\left\{\|\tilde{b}-A x\|^{2}+\alpha\|L x\|^{2}\right\}, \quad \alpha>0,
$$

see, e.g., Groetsch. ${ }^{6}$ Here and in the sequel $\|\cdot\|$ denotes the Euclidean vector norm. The matrix $L$ is referred to as a regularization operator and the term $\alpha\|L x\|^{2}$ in (5) penalizes solutions whose image under $L$ have large norm. The regularization operator $L$ is often chosen to be the $m^{2} \times m^{2}$ identity matrix or a discrete approximation of a derivative operator. Minimization problems of the form (5) are referred to as penalized least-squares problems. It can be shown that the solution $\tilde{x}_{\alpha}$ of (5) satisfies the linear system of equations

$$
\left(A^{T} A+\alpha^{1 / 2} L^{T} L\right) \tilde{x}_{\alpha}=A^{T} \tilde{b} .
$$

Thus, Tikhonov regularization replaces the linear system (4) by the linear system (6). The regularization parameter $\alpha$ controls how much the regularized solution $\tilde{x}_{\alpha}$ differs from the solution $\tilde{x}$ of (4), since $\tilde{x}_{\alpha} \rightarrow \tilde{x}$ as $\alpha \searrow 0$. Choosing an appropriate value of $\alpha$ is an important part of the Tikhonov regularization method. However, this topic is outside the scope of the present paper.

Tikhonov regularization can produce poor regularized solutions when the desired solution $x$ comes from an image with edges, i.e., in situations when the function $f$ in (1) contains discontinuities. In particular, images corresponding to Tikhonov regularized solutions often exhibit "ringing" or "halo" artifacts near "sharp" edges. The total variation (TV)-penalized Tikhonov method addresses this problem by replacing the penalty term $\|L x\|^{2}$ in (5) by a discrete approximation of the total variation functional

$$
J_{T V}(f)=\int_{\Omega}\left(\left(\frac{\partial f}{\partial \xi}\right)^{2}+\left(\frac{\partial f}{\partial \eta}\right)^{2}\right)^{1 / 2} d \xi d \eta
$$

cf. equation (1). The total variation functional does not penalize discontinuities in the sought-after regularized solution. Thus, TV-penalized Tikhonov regularization is an attractive regularization method for image restoration problems when the noise- and blur-free image sought has edges. This regularization method has received considerable attention, see, e.g., Vogel and Oman ${ }^{15}$ and references therein. An example of TV-penalized Tikhonov regularization is shown in Section 4.

Computing the TV-penalized Tikhonov regularized solution can be expensive. This drawback can be a significant one for image restoration problems, because the resulting linear systems (4) tend to be large. The application of a few steps of a Krylov iterative method provides a more computationally efficient approach to the determination of a regularized solution of (4). This paper compares the performance of several Krylov subspace iterative methods based on the GMRES method with that of TV-penalized Tikhonov regularization for 
restoration of images with "sharp" edges that have been degraded by noise and blur. The GMRES method is a well-known Krylov subspace iterative method for the solution of linear systems of equations with a nonsingular and nonsymmetric matrix; see, e.g., Saad and Schultz ${ }^{14}$ or Saad. ${ }^{13}$ The application of the GMRES and related methods to ill-posed problems and image restoration problems has been discussed previously by Calvetti et al.. ${ }^{3-5}$

When applying a few steps of a Krylov subspace iterative method to the linear system (4), the number of steps plays the role of the regularization parameter $\alpha$. We denote the regularized solution defined by taking $\ell$ steps of a Krylov subspace iterative method applied to the linear system (4) by $\tilde{x}_{\ell}$.

\section{THE GMRES AND RRGMRES METHODS}

This section reviews properties of the GMRES method by Saad and Schultz ${ }^{14}$ and of the range restricted GMRES (RRGMRES) method introduced by Calvetti et al.. ${ }^{2}$

Introduce the Krylov subspaces

$$
\mathcal{K}_{\ell}(A, \tilde{b})=\operatorname{span}\left\{\tilde{b}, A \tilde{b}, \ldots, A^{\ell-1} \tilde{b}\right\}, \quad \ell=1,2,3 \ldots .
$$

Let the initial approximate solution be $\tilde{x}_{0}=0$ and denote the approximate solution determined by applying $\ell \geq 1$ steps of the GMRES method to the linear system (4) by $\tilde{x}_{\ell}$. Then $\tilde{x}_{\ell}$ satisfies

$$
\left\|A \tilde{x}_{\ell}-\tilde{b}\right\|=\min _{\tilde{x} \in \mathcal{K}_{\ell}(A, \tilde{b})}\|A \tilde{x}-\tilde{b}\|, \quad \tilde{x}_{\ell} \in \mathcal{K}_{\ell}(A, \tilde{b}) .
$$

Algorithm 2.1 below describes an implementation of the GMRES method based on the Arnoldi process. The computation of the iterate $\tilde{x}_{\ell}$ by Algorithm 2.1 requires $\ell$ evaluations of matrix-vector products with the matrix $A$.

It follows from (9) that we can express the regularized solution produced by $\ell$ steps of the GMRES method as

$$
\tilde{x}_{\ell}=\gamma_{1} \tilde{b}+\gamma_{2} A \tilde{b}+\cdots+\gamma_{\ell} A^{\ell-1} \tilde{b}
$$

for certain scalars $\gamma_{j}, 1 \leq j \leq \ell$. The inclusion of a multiple of the noisy right-hand side $\tilde{b}$ in the approximate solutions $\tilde{x}_{\ell}, \ell=1,2,3, \ldots$, determined by the GMRES method can give rise to "noisy" restorations.

The Range Restricted GMRES (RRGMRES) method, proposed by Calvetti et al., ${ }^{2}$ is a variant of the GMRES method. Let the initial iterate be given by $\tilde{x}_{0}=0$. Then the iterates $\tilde{x}_{\ell}, \ell \geq 1$, determined by the RRGMRES method satisfy

$$
\left\|A \tilde{x}_{\ell}-\tilde{b}\right\|=\min _{\tilde{x} \in \mathcal{K}_{\ell}(A, A \tilde{b})}\|A \tilde{x}-\tilde{b}\|, \quad \tilde{x}_{\ell} \in \mathcal{K}_{\ell}(A, A \tilde{b}),
$$

where

$$
\mathcal{K}_{\ell}(A, A \tilde{b})=\operatorname{span}\left\{A \tilde{b}, A^{2} \tilde{b}, \ldots, A^{\ell} \tilde{b}\right\}, \quad \ell=1,2,3 \ldots .
$$

The RRGMRES method was developed for the solution of inconsistent linear systems of equations with a nonsymmetric matrix. The RRGMRES method requires one more matrix-vector product evaluation with the matrix $A$ than the GMRES method and the same storage to evaluate the $\ell$ th iterate. An implementation of the RRGMRES method is presented by Algorithm 2.1 below. We remark that the Krylov subspaces used by the RRGMRES method lack the noisy right-hand side vector $\tilde{b}$ and the images corresponding to the regularized solutions produced by the RRGMRES method tend to be less noisy than those produced by the GMRES method.

The following algorithm presents implementations based on the Arnoldi process of both the GMRES and RRGMRES methods. The algorithm appeared in Calvetti et al. ${ }^{4}$ and is repeated here for the reader's convenience. The GMRES implementation is due to Saad and Schultz. ${ }^{14}$ 
Input: $A, \tilde{b}, \ell, M E T H O D \in\{R R G M R E S, G M R E S\}$.

Output: Approximate solution $\tilde{x}_{\ell}$ of (4).

if $M E T H O D=G M R E S$

$v_{1}:=\tilde{b} /\|\tilde{b}\|$,

elseif $M E T H O D=R R G M R E S$

$v_{1}:=A \tilde{b} /\|A \tilde{b}\|$,

endif

Define $\bar{H}_{\ell}:=\left[h_{i j}\right], h_{i j}:=0,1 \leq i \leq \ell+1,1 \leq j \leq \ell$,

for $j=1,2, \ldots, \ell d o$

$w:=A v_{j}$,

for $i=1,2, \ldots, j$ do

$h_{i j}:=v_{i}^{T} w, w:=w-h_{i j} v_{i}$,

end

$h_{j+1, j}:=\|w\|, v_{j+1}:=w / h_{j+1, j}$,

end

$V_{\ell}:=\left[v_{1}, v_{2}, \ldots, v_{\ell}\right], V_{\ell+1}:=\left[v_{1}, v_{2}, \ldots, v_{\ell+1}\right]$,

$\tilde{y}_{\ell}:=\bar{H}_{\ell}^{\dagger} V_{\ell+1}^{T} \tilde{b}$,

$\tilde{x}_{\ell}:=V_{\ell} \tilde{y}_{\ell}$

Algorithm 2.1 breaks down if $h_{j+1, j}=0$. It can be shown that breakdown will occur (in exact arithmetic) for some iteration index $j \leq m^{2}$, and that $A \tilde{x}_{j}=\tilde{b}$ when this occurs, i.e., $\tilde{x}_{j}=\tilde{x}$. Since $\tilde{x}$ is generally a poor approximation of the solution $x$ of (2), the algorithm is usually stopped well before breakdown occurs. We assume in the remainder of this paper that the regularized solutions produced by the GMRES or RRGMRES methods, as implemented by Algorithm 2.1, are determined by terminating the iterations before breakdown.

We remark that the columns of the matrix $V_{\ell}=\left[v_{1}, v_{2}, \ldots, v_{\ell+1}\right]$ produced by Algorithm 2.1 form an orthonormal basis of the Krylov subspaces $\mathcal{K}_{\ell+1}(A, \tilde{b})$ for the GMRES method, or of $\mathcal{K}_{\ell+1}(A, A \tilde{b})$ for the RRGMRES method.

Note that the blurring matrix $A$ in (2) and (4) corresponding to image restoration problems of the form (1) typically exhibit structure that makes rapid evaluation of matrix-vector products possible; see, e.g., Jain ${ }^{12}$ for a discussion.

\section{THE HYBRID GMRES METHOD}

In Section 1, we discussed the use of a TV penalty functional in the minimization problem (5) to help produce good restorations of images that have been degraded by noise and blur. In this section, we present an extension of the GMRES method, that adds a penalty term that approximates a total variation functional of $f$ in (1) on a subspace of small dimension. We refer to this hybrid method as the GMRES+ method. The extension is inspired by the PP-TSVD method proposed by Hansen and Mosegaard. ${ }^{9-11}$ It can also be applied be applied to the RRGMRES method.

Let $\tilde{x}_{\ell}$ be the regularized solution of (4) produced by the GMRES method after $\ell$ steps of Algorithm 2.1. Then the $m^{2} \times m^{2}$ matrix

$$
A_{\ell}=V_{\ell+1} \bar{H}_{\ell} V_{\ell}^{T},
$$


where $V_{\ell+1}, V_{\ell}$, and $\bar{H}_{\ell}$ are defined in Algorithm 2.1, can be thought of as a rank- $\ell$ approximation of the matrix $A$ with a well-defined null space of dimension $m^{2}-\ell$. Let $1 \leq n \leq m^{2}-\ell$ and assume that $\ell+n$ steps of Algorithm 2.1 can be carried out without break down. It follows from the definition of $A_{\ell}$ and Algorithm 2.1 that the columns of the $m^{2} \times n$ matrix

$$
V^{0}=\left[v_{\ell+1}, v_{\ell+2}, \ldots, v_{\ell+n}\right]
$$

are in the null space of $A_{\ell}$. The GMRES+ method solves the minimization problem

$$
\min _{z \in \mathbf{S}}\|L z\|_{p} \text { subject to }\left\|A_{\ell} z-\tilde{b}\right\|=\min , \quad 1 \leq p \leq 2,
$$

where $\|\cdot\|_{p}$ denotes the $\ell_{p}$-norm and $\mathbf{S} \subset \mathbf{R}^{m^{2}}$ is the affine subspace defined by $\left\{\tilde{x}_{\ell}+V^{0} y: y \in \mathbf{R}^{n}\right\}$. It follows from (11), the definition of $A_{\ell}$, and (9), that $\left\|A_{\ell} z-\tilde{b}\right\|$ has the same value for all $z \in \mathbf{S}$. Thus, we can rewrite the $\ell_{p}$-minimization problem in (12) as

$$
\min _{y \in \mathbf{R}^{n}}\left\|L\left(\tilde{x}_{\ell}+V^{0} y\right)\right\|_{p}=\min _{y \in \mathbf{R}^{n}}\left\|L \tilde{x}_{\ell}+L V^{0} y\right\|_{p} .
$$

This is an unconstrained $\ell_{p}$-minimization problem and can be solved in a variety of ways, e.g., by the iteratively reweighted least-squares (IRLS) method, see, e.g., Björck ${ }^{1}$ for a discussion and references.

We now turn to the construction of a suitable regularization operator $L$ for use in (12). A widely used approximation of the total variation functional $J_{T V}$ defined by (7) is given by

$$
J(f)=\int_{\Omega}\left(\left|\frac{\partial f}{\partial \xi}\right|+\left|\frac{\partial f}{\partial \eta}\right|\right) d \xi d \eta
$$

Introduce the discrete approximation of the first derivative operator

$$
L_{1}=\left[\ell_{j, k}\right]=\left(\begin{array}{cccc}
-1 & 1 & & \\
& \ddots & \ddots & \\
& & -1 & 1
\end{array}\right) \in \mathbf{R}^{(m-1) \times m}
$$

and define the $\left(2 m^{2}-2 m\right) \times m^{2}$ matrix $L$ by

$$
L=\left(\begin{array}{c}
L_{1} \otimes I \\
I \otimes L_{1}
\end{array}\right)
$$

where $I$ denotes the $m \times m$ identity matrix, and $L_{1} \otimes I$ denotes the Kronecker product of $L_{1}$ and $I$ given by the $(m-1) m \times m^{2}$ matrix

$$
L_{1} \otimes I=\left(\begin{array}{cccc}
\ell_{1,1} I & \ell_{1,2} I & \cdots & \ell_{1, m} I \\
\ell_{2,1} I & \ell_{2,2} I & \cdots & \ell_{2, m} I \\
\vdots & \vdots & \vdots & \vdots \\
\ell_{m-1,1} I & \ell_{m-1,2} I & \cdots & \ell_{m-1, m} I
\end{array}\right)
$$

Let the vector $x=\left(x_{1}, x_{2}, \ldots, x_{m^{2}}\right)^{T}$ represent the row-ordered discretization of the function $f$ as outlined in Section 1. Up to a scaling factor,

$$
\|L x\|_{p}
$$

provides an approximation of $J(f)$ for $p$ close to 1 ; see Hansen. ${ }^{9}$ In the computed examples presented in Section 4 , we solve the minimization problem (12) with $p=1.1$. We remark that other approximations of the total variation functional also can be used.

We are now ready to present an algorithm for the GMRES+ method.

AlgORIthm 3.1. The GMRES+ method 
Input: $A, \tilde{b}, \ell, n$.

Output: Approximate solution $\tilde{x}_{\ell}$ of (4).

Compute the regularized solution $\tilde{x}_{\ell}$ produced by $\ell$ steps of the GMRES method (Algorithm 2.1).

Continue Algorithm 2.1 up to step $\ell+n$.

Compute the minimizer $\tilde{y}$ of (13) or (12) with $L$ defined by (15).

$\tilde{x}_{\ell}:=\tilde{x}_{\ell}+V^{0} \tilde{y}$, where $V^{0}$ is defined by (11).

We remark that the structure of the matrix $L$ in $(15)$ can be exploited to efficiently compute matrix-vector products involving $L$. Moreover, although the matrix $V^{0}$ is dense, the $\ell_{p}$-minimization problem (13) remains computationally manageable for small values of $n$.

\section{NUMERICAL EXPERIMENTS}

We consider the restoration of a noisy and blurred test image consisting of a disc with a uniform background. Although the test image contains an edge, the overall contrast in the image is low, i.e., the difference in brightness between the disc and the background is low. The noise- and blur-free test image consists of a $256 \times 256$ regular mesh of grayscale pixel values in the range $[0,255]$. Thus $m=256$ in (2) and (4). The noise vector $d$ in (3) is constructed as follows: Let the entries of the vector $x$ contain the pixel values of the noise- and blur-free model image ordered row-wise and let $A$ represent the blurring matrix. Then the vector $b=A x$ represents the blurred, but noise-free, image associated with the model image represented by $x$. The noise vector $d \in \mathbf{R}^{m^{2}}$ has normally distributed random entries scaled so that $\|d\|=0.01\|A x\|$. The nonsymmetric blurring matrix $A$ is constructed from an empirically determined point spread function that models atmospheric blur. The blurring matrix is from the Image Deblurring package for Matlab by Hamilton and Vogel, available at the web site http://www.math.montana.edu/ ${ }^{\sim}$ vogel/Software/deconv. The original (unknown) blur- and noise-free image and the blurred and noisy image to be restored are displayed in Figure 1.

We compare the GMRES, GMRES+ and RRGMRES methods applied to the linear system (4) with the TV-penalized Tikhonov regularization method. We used the initial approximate solution $x_{0}=0$ for the GMRESbased methods.

All computations were carried out using Matlab 5.3.1 on an Intel Pentium workstation with about 16 significant decimal digits. Computations for the TV-penalized Tikhonov regularization used the Image Deblurring package for Matlab mentioned above. This package uses a quasi-Newton method to solve the minimization problem (5) with a total variation penalty functional in place of $\|L x\|^{2}$ for fixed $\alpha$. Each step of the quasi-Newton method requires the solution of a linear system of equations with $m^{2}$ unknowns and a sparse, symmetric positive definite matrix. A preconditioned conjugate gradient method with a preconditioner based on multilevel splitting is used to solve this linear system.

We were able to compute the relative error norms $\left\|\tilde{x}_{\ell}\right\| /\|x\|$ and $\left\|\tilde{x}_{\alpha}\right\| /\|x\|$ for the GMRES-based and TVpenalized Tikhonov methods since the blur- and noise-free image, represented by $x$, is available. In each case, we chose the termination index $\ell$ for the GMRES-based methods so that the computed solution $\tilde{x}_{\ell}$ exhibits the smallest relative error. We chose the value $\alpha_{1}=1.0 \cdot 10^{-12.5}$ of the regularization parameter $\alpha$ in the TVpenalized Tikhonov regularization method; this value gives the smallest, or close to the smallest, relative error. We also present results for a value $\alpha_{2}=1.0 \cdot 10^{-12.0}$ of the regularization parameter; the latter value yields a relative error only slightly larger than that obtained with $\alpha_{1}$. The TV-penalized Tikhonov regularized solution $\tilde{x}_{\alpha_{2}}$ was produced with less computational work than the regularized solution $\tilde{x}_{\alpha_{1}}$. We let $n=\ell$ in the GMRES+ method.

Table 1 compares the number of matrix-vector products with the matrices $A$ or $A^{T}$ required for each method. The evaluation of these matrix-vector products constitutes the bulk of the floating point work for the GMRES, RRGMRES and TV-penalized Tikhonov regularization methods. The solution of the $\ell_{p}$-minimization problem by the IRLS method required by the GMRES+ method involved extra computational work, which amounted 
to the equivalent of approximately 5 additional matrix-vector product evaluations. This extra work was added to the number of matrix-vector products for the GMRES+ method in Table 1. The table shows that all of the GMRES-based methods required significantly less computational work than the TV-penalized Tikhonov method.

Figure 2 shows the restored images produced by each method. All of the methods do a good job of restoring the image, but the solution produced by the GMRES method clearly exhibits a great deal more noise than the other restored images. However, the GMRES method also required the least amount of computational work. The GMRES + method improves the image produced by the GMRES method significantly, as can be seen by visual comparison of the restored images, and by the values of the relative error norms reported in Table 1 . The RRGMRES method produced the best qualitative visual restoration in this example.

Computational experience from numerous image restoration problems indicates that the GMRES+ method often gives a less noisy, and therefore more pleasing, restorations than the GMRES method. How well the GMRES+ method performs depends on how appropriate the space $\mathbf{S}$ is. For some problems, such as the one of the present paper, the RRGMRES method also gives pleasing restorations. A careful comparison of various GMRES based methods for image restoration still has to be done. In our experience, all GMRES based methods require significantly fewer matrix-vector product evaluations than the TV-penalized Tikhonov regularization method described by Vogel and Oman. ${ }^{15}$

\begin{tabular}{|l|ccccc|}
\hline & GMRES & GMRES+ & RRGMRES & TV $\left(\alpha_{1}\right)$ & TV $\left(\alpha_{2}\right)$ \\
\hline Number of iterations & 3 & 6 & 11 & - & - \\
Matrix-vector products & 3 & $11^{*}$ & 12 & 90 & 54 \\
Relative error norm & $7.98 \cdot 10^{-2}$ & $6.60 \cdot 10^{-2}$ & $4.18 \cdot 10^{-2}$ & $4.13 \cdot 10^{-2}$ & $4.28 \cdot 10^{-2}$ \\
\hline
\end{tabular}

Table 1. Summary of numerical results: Number of iterations is counted only for the GMRES-based methods. Matrixvector products shows the total number of matrix-vector product evaluations with the matrices $A$ and $A^{T}$. The relative error norm is defined by $\left\|x-\tilde{x}_{\alpha}\right\| /\|x\|$, where $\tilde{x}_{\alpha}$ is the regularized approximate solution produced by the respective method. ${ }^{*}$ The number of matrix-vector products for the GMRES+ method was increased by 5 to reflect the extra work required by the IRLS method. The values of the regularization parameter for the TV-penalized Tikhonov examples are $\alpha_{1}=1.0 \cdot 10^{-12.5}$ and $\alpha_{2}=1.0 \cdot 10^{-12.0}$.
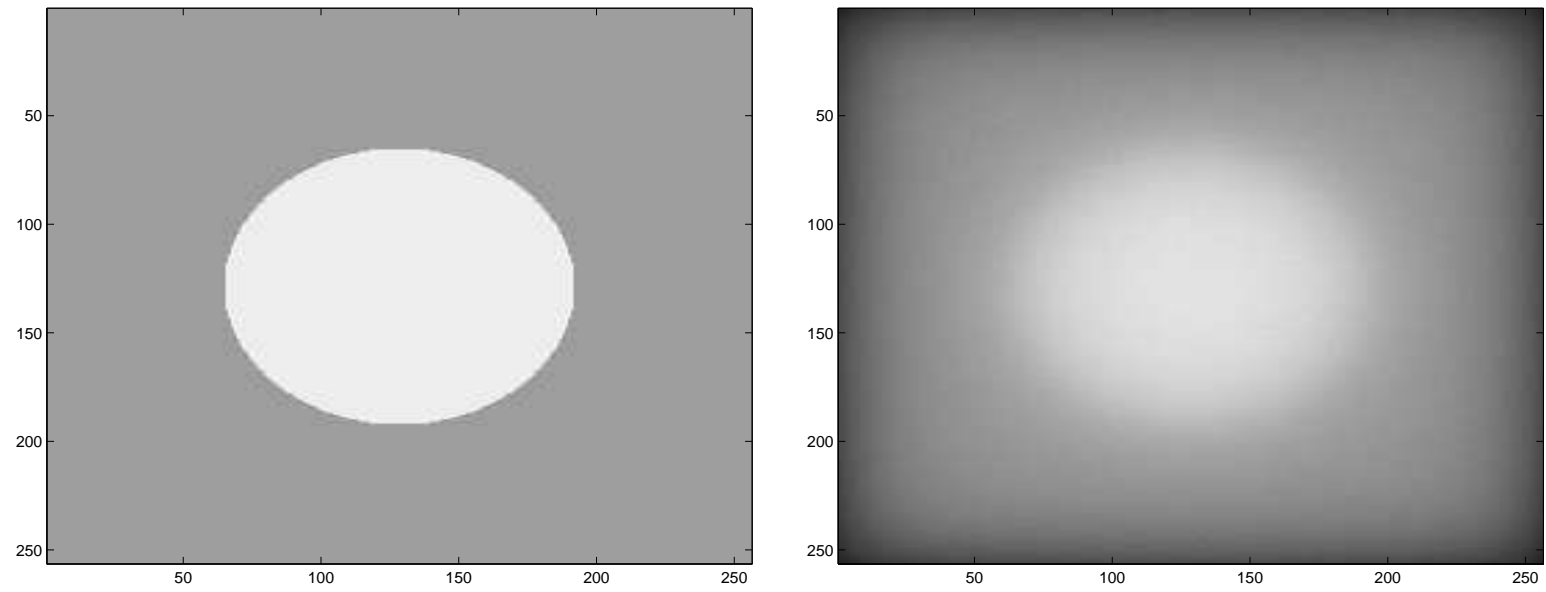

Figure 1. Original (unknown) blur- and noise-free image (left), available blurred and noisy image to be restored (right).

\section{REFERENCES}

1. Å. Björck, Numerical Methods for Least Squares Problems, SIAM, Philadelphia, 1996.

2. D. Calvetti, B. Lewis and L. Reichel, GMRES-type methods for inconsistent systems, Linear Algebra Appl., 316 (2000), pp. 157-169. 
3. D. Calvetti, B. Lewis and L. Reichel, On the regularizing properties of the GMRES method, Numer. Math., 91 (2002), pp. 605-625.

4. D. Calvetti, B. Lewis and L. Reichel, Restoration of images with spatially variant blur by the GMRES method, in Advanced Signal Processing Algorithms, Architectures, and Implementations X, ed. F.T. Luk, Proceedings of the Society of Photo-Optical Instrumentation Engineers (SPIE), vol. 4116, The International Society for Optical Engineering, Bellingham, WA, 2000, pp. 364-374.

5. D. Calvetti, B. Lewis and L. Reichel, On the choice of subspace for iterative methods for linear discrete ill-posed problems, Int. J. Appl. Math. Comput. Sci., 11 (2001), pp. 1069-1092.

6. C. W. Groetsch, The Theory of Tikhonov Regularization for Fredholm Equations of the First Kind, Pitman, Boston, 1984.

7. P. C. Hansen, Regularization tools: A Matlab package for analysis and solution of discrete ill-posed problems, Numer. Algorithms, 6 (1994), pp. 1-35.

8. P. C. Hansen, Rank-Deficient and Discrete Ill-Posed Problems, SIAM, Philadelphia, 1998.

9. P. C. Hansen, The PP-TSVD algorithm for image restoration problems, in Methods and Applications of Inversion, eds. P. C. Hansen, B. H. Jacobsen and K. Mosegaard, Lecture Notes in Earth Sciences 92, Springer, Berlin, 2000.

10. P. C. Hansen and K. Mosegaard, Piecewise polynomial solutions to linear inverse problems, in Lecture Notes in Earth Sciences 63, eds. B. H. Jacobsen, K. Mosegaard and P. Sbani, Inverse Methods, Springer, Berlin, 1996.

11. P. C. Hansen and K. Mosegaard, Piecewise polynomial solutions without a priori break points, Num. Lin. Alg. Appl., 3 (1996), pp. 513-524.

12. A. K. Jain, Fundamentals of Digital Image Processing, Prentice-Hall, Englewood Cliffs, New Jersey, 1989.

13. Y. Saad, Iterative Methods for Sparse Linear Systems, PWS, Boston, 1996.

14. Y. Saad and M. H. Schultz, GMRES: a generalized minimal residual method for solving nonsymmetric linear systems, SIAM J. Sci. Stat. Comput., 7 (1986), pp. 856-869.

15. C. R. Vogel and M. E. Oman, Fast, robust total variation-based reconstruction of noisy, blurred images, IEEE Transactions on Image Processing, 7 (1998), pp. 813-824. 

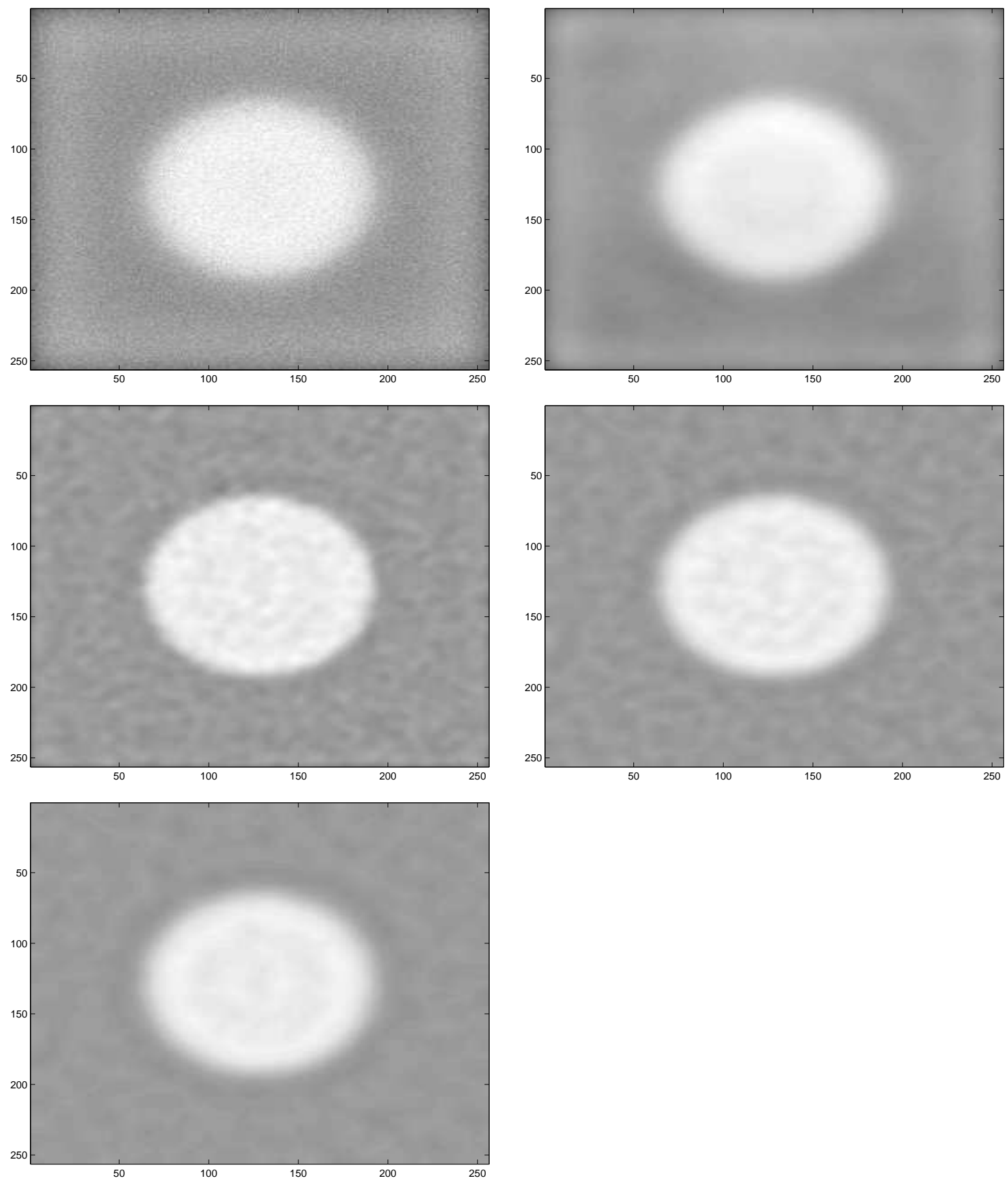

Figure 2. Restored images: GMRES (top left), GMRES+ (top right), RRGMRES (middle left), TV-penalized Tikhonov with $\alpha=\alpha_{1}$ (middle right), TV-penalized Tikhonov with $\alpha=\alpha_{2}$ (lower left). 